\title{
Statistical Modeling of MicroRNA Expression with Human Cancers
}

\section{Ke-Sheng Wang ${ }^{1 *}$, Yue Pan ${ }^{2}$ and Chun $\mathrm{Xu}^{3}$}

${ }^{1}$ Department of Biostatistics and Epidemiology, College of Public Health, East Tennessee State University, Johnson City, TN, USA

${ }^{2}$ Department of Public Health Sciences, Miller School of Medicine, University of Miami, Miami, FL, USA

${ }^{3}$ Department of Pediatrics, Paul L. Foster School of Medicine, Texas Tech University Health Sciences Center, EI Paso, TX, USA

\begin{abstract}
MicroRNAs (miRNAs) are small non-coding RNAs (containing about 22 nucleotides) that regulate gene expression. MiRNAs are involved in many different biological processes such as cell proliferation, differentiation, apoptosis, fat metabolism, and human cancer genes; while miRNAs may function as candidates for diagnostic and prognostic biomarkers and predictors of drug response. This paper emphasizes the statistical methods in the analysis of the associations of miRNA gene expression with human cancers and related clinical phenotypes: 1) simple statistical methods include chi-square test, correlation analysis, t-test and one-way ANOVA; 2) regression models include linear and logistic regression; 3) survival analysis approaches such as non-parametric Kaplan-Meier method and log-rank test as well as semi-parametric Cox proportional hazards models have been used for time to event data; 4) multivariate method such as cluster analysis has been used for clustering samples and principal component analysis (PCA) has been used for data mining; 5) Bayesian statistical methods have recently made great inroads into many areas of science, including the assessment of association between miRNA expression and human cancers; and 6) multiple testing.
\end{abstract}

Keywords: Cancer; MicroRNA; MiRNA; Biomarker; Diagnosis; Regression Models; Non-parametric Methods; Survival Analysis; Multivariate Statistics; Bayesian Methods; Multiple Testing

\section{Background}

A microRNA (miRNA) is a small non-coding RNA molecule (containing about 22 nucleotides), which functions in RNA silencing and post-transcriptional regulation of gene expression [1-3]. MiRNAs are involved in many different biological processes such as cell proliferation, differentiation, apoptosis, fat metabolism, and human cancer genes [4-8]. Previous studies suggest that miRNAs expression profiles are correlated with disease pathogenesis and prognosis, and may ultimately be useful in the management of human cancer; while miRNAs may function as candidates for diagnostic and prognostic biomarkers and predictors of drug response. For example, miRNAs can act either as oncogenes or tumor suppressors contributing to initiation and progression of cancer [3,6,9-12]. MiRNA expression can be detected by a two-step polymerase chain reaction process of RT-PCR followed by quantitative PCR [13], microarray [14], and miRNA sequencing [15]. The raw counts of miRNA expression are usually skewed and not meet the assumptions of parametric statistical tests, therefore a $\log 2$ transformation is applied [16,17], for example by a weighted trimmed mean of the log expression ratios using the $\mathrm{R} /$ Bioconductor package $[18,19]$.

\section{Simple Statistical Methods}

Some studies examined the miRNA expression level as a categorical variable, which was dichotomized as low and high level expression based on the median [20-22]. The chi-square test has been used to determine the associations of expression of miRNA-21 with patients' clinical parameters such as lymph node metastasis, clinical stage and poor prognosis in non-small cell lung cancer (NSCLC) as categorical variables [22]. This approach was also used to test the relationship between miRNA-221 expression and binary clinicopathologic features such as histology, p-TNM stage and history of smoking [23]; and the correlation between miRNA-124 down-regulates SOX8 expression and binary clinicopathologic factors such as tumor size, lymph node metastasis, differentiation classification and clinical stage [24].

However, most other studies treated the miRNA expression as a continuous variable. For example, the t-test for two-independent samples has been used to compare the expression values of miRNAs between the control and lung cancer groups [17,25], the expression of miRNA-146 in NSCLC cancer tissue (43 individuals) and normal tissue (32 individuals) [26]. Furthermore, the t-test for paired samples was performed to identify if miRNA-31 is differentially expressed between lung adenocarcinoma and matched normal adjacent tissues using the Cancer Genome Atlas (TCGA) dataset [27]. Moreover, oneway ANOVA was utilized to examine the association between E2F miRNA expression and degree of tumor cell invasion in gastric cancer [28]. Additionally, non-parametric methods such as Wilcoxon signrank test for paired samples analysis was used to compare expression of miRNAs across the seven tumor samples in breast cancer [29] and Kruskal-Wallis test was used to determine the significance of miRNAs among the biochemical failure categories [30].

The simple correlation analysis has been used to describe the relationship of miRNA expression with cancer clinical phenotypes. For example, the correlation between miRNA-148a expression and the methylation level of the DNA region encoding miRNA-148a in tissues was evaluated by Pearson's correlation [31]. Other studies used the Spearman's rank correlation analysis to correlate miRNAs with mRNA in the breast cancer samples [32], the alterations of methylation with expression level of miRNA-9-1 gene in the lung tumors [33], and the pairwise miRNA expression in seven types of cancer using the TCGA data [34].

\section{Linear and Logistic Regression Models}

The linear and logistic regression models have been used to

*Corresponding author: Ke-Sheng Wang, Department of Biostatistics and Epidemiology, College of Public Health, East Tennessee State University, Johnson City, TN, USA, Tel: +1 423439 4481; Fax: +1 423439 4606; E-mail: wangk@etsu.edu

Received July 06, 2015; Accepted July 14, 2015; Published July 21, 2015

Citation: Wang KS, Pan Y, Xu C (2015) Statistical Modeling of MicroRNA Expression with Human Cancers. J Biom Biostat 6: 240. doi:10.4172/21556180.1000240

Copyright: $\odot 2015$ Wang KS, et al. This is an open-access article distributed under the terms of the Creative Commons Attribution License, which permits unrestricted use, distribution, and reproduction in any medium, provided the original author and source are credited. 
examine the associations of miRNA expression with cancer and clinical phenotypes. For example, linear regression analysis was used to identify the association of 335 miRNAs with recurrent ovarian cancer cell lines [35] and to examine the association between cigarettes per day in lung cancer patients and miRNA expression [36]. Recently, a multiple linear regression analysis was used to investigate the associations of each mRNA transcript with the corresponding miRNAs in colorectal cancer [37].

The binary logistic regression analysis was utilized to select the top 25 miRNA genes with prognostic relevance in neuroblastoma, that is, the miRNAs with the lowest P-value in a model testing miRNA expression levels (below or above the median expression) versus overall survival [38], evaluate the ability of chosen miRNAs to distinguish between breast cancer cases and controls [39], and differentially expressed genes that are regulated by the mRNAs expression between gastric cancer and paired adjacent normal tissues [28]. Multiple logistic regression analyses have been performed to investigate the role of miRNA signature on binary outcomes in neuroblastoma such as cases defined as relapse, progression, or death from disease (progressionfree survival), and death (overall survival) comparing with controls defined as nonfailure in the first 3 years of follow-up, adjusted for age at diagnosis, stage and other phenotypes [38]. Another study used a multiple logistic model of specific miRNA biomarkers to predict the presence of menstrual blood [40]. Recently, Zhang et al. [41] evaluated the associations of both human papillomavirus-HPV16 status and IL-1 $\alpha$ rs3783553 polymorphism at the miRNA-122 binding site, individually and in combination, with the risk of oral squamous cell carcinoma (OSCC) using both univariate and multivariable logistic regression analyses.

\section{Survival Analysis}

The non-parametric Kaplan-Meier method has been used to estimate the survival distributions for expression of miRNA-21 [42], miRNA-148a [31], miRNA-31 [27], miRNA-221 [23], and miRNA-124 in NSCLC [24]; while the log-rank test has been used to analyze the survival differences according to expression between different subgroups [23,24,27,31,42]. Recently, the Kaplan-Meier method was used to estimate the overall survival curves for four miRNAs (miRNA-21, 22, 155 and 210) in the TCGA glioblastoma multiforme dataset using the BRB-Array Tools - R/BioConductor [16]. Another recent study used the Kaplan-Meier survival analysis to estimate the survival distributions for patients in each of 5 subtypes of glioblastoma multiforme based on significant miRNAs and applied the log-rank test to assess the statistical significance between the stratified high- and low-risk survival groups in primary glioblastoma in the TCGA dataset using GraphPad Prism 6.0 statistical software [43].

The semi-parametric Cox proportional hazards model has been widely used to construct respective prognostic miRNA signatures of cancers $[21,22,27,42,43]$. For example, multivariable Cox proportional hazards model was used to identify the significant influence of miRNA148a expression on survival, adjusted for gender, age, histologic grade, $\mathrm{T}$ stage and smoking status [31]. Another study used the univariable Cox regression analysis to examine the association between 9 continuous miRNA expression and overall survival rate of Glioblastoma Multiforme (GBM) using the TCGA dataset; while multivariable Cox regression analysis was performed by stratifying patients according to age and using MGMT methylation status, IDH1 mutations, pre-treatment, recurrence, and TCGA prognostic classification as covariates [16]. Especially, Mankoo et al. [44] implemented a multivariate Cox Lasso model and median time-to-event prediction algorithm and applied it to two datasets integrated from the four genomic data types (mRNA, DNA methylation, DNA copy-number alteration and miRNA) in the TCGA data.

\section{Multivariate Methods}

Multivariate analysis methods such as cluster analysis has been used for clustering samples, and principal component analysis (PCA) has been used for data mining. For example, to identify miRNA expression patterns in breast cancer, one study performed an unsupervised hierarchical complete linkage cluster analysis using Euclidean distance as dissimilarity metric; meanwhile a Pearson $\chi^{2}$ test and a Goeman global test were used to investigate the association of the sample clustering result with clinicopathological variables $[32,45]$. Keller et al. [25] analyzed the abundance of 904 miRNAs in serum from eight cancer patients at three time points and from six healthy control individuals and identified clusters based on the identified miRNA signatures, hierarchical clustering and a self-organizing map; while Oberg et al. [46] used PCA and hierarchical cluster analyses to visualize miRNA expression patterns present at a global level in colon cancer. Another study applied a complete linkage hierarchical clustering using the Euclidian distance to compute the dissimilarity of miRNA and samples independently of each other using the normalized data [17]. In addition, $\mathrm{Li}$ et al. [21] identified an eight-miRNA (miR-31, miR196b, miR-766, miR-519a-1, miR-375, miR-187, miR-331 and miR101-1) signature for the prediction of overall survival of patients with lung adenocarcinoma (LUAD) using supervised principal components method in the TCGA-derived LUAD cohort.

\section{Bayesian Statistics}

Bayesian statistical methods have recently made great inroads into many areas of science [47], including the assessment of association between miRNA expression and human cancers. One of the main challenges in modeling the statistical dependence between highthroughput studies is that a large number of measurements (usually in thousands) is available for a relatively small number (usually in tens or hundreds) of patient samples; therefore, classical statistical approaches based on linear models and hierarchical clustering are prone to overfitting [48,49]. In these situations, Efron [48] recommended accounting for high-dimensionality by using approaches that borrow information across covariates to compensate for the limited information available across samples, which leads to better and more reliable inference.

Several approaches have been developed to address these challenges in various contexts, for example hierarchical Bayesian modeling approaches based on linear shrinkage estimators [50]. For predicting relevant clinical outcomes, Srivastava et al. [51] proposed a flexible statistical machine learning approach that acknowledges and models the interaction between platform-specific measurements through nonlinear kernel machines and borrows information within and between platforms through a hierarchical Bayesian framework. The methods of integrating gene/mRNA expression and miRNA profiles for predicting patient survival times were applied to the TCGA based glioblastoma multiforme (GBM) dataset [51]. Recently, Chekouo et al. [52] proposed a novel Bayesian model to identify miRNAs and their target genes that were associated with survival time by incorporating the miRNA regulatory network through prior distributions.

\section{Multiple Testing}

The number of miRNA in human genome is abundant and multiple 
outcomes have been measured in many miRNA expression studies. Therefore, the multiple testing issue is a big statistical challenge. The conservative Bonferroni correction $(0.05 / \mathrm{n}, \mathrm{n}$ is the number of tested miRNA) has been used for dealing with multiple testing [53]. Furthermore, the false discovery rate (FDR) based on the Benjamini and Hochberg method [54] was used to correct multiple testing in comparison of miRNA expression levels across different groups [5557]. Additionally, some studies have used permutation tests to account for multiple testing in comparison of miRNA expression profile, for example, by using 1,000 or 10,000 permutations $[36,43,53]$.

\section{Discussion and Future Direction}

The t-test and one-way ANOVA require the assumption of normality of the data; however, such assumption is often violated in practice. Therefore, generalized linear models (GLMs) and generalized linear mixed models (GLMMs) can relax the assumption and deal with binary and counts data. For example, GLMMs can be used to compare the miRNA measures before/after or with/without treatment. Furthermore, non-parametric statistical methods may be alternatives in some specific situations to describe the miRNA data with cancer.

In survival analysis, many studies have focused on non-parametric methods such as Kaplan-Meier method and log-rank test and semiparametric Cox proportional hazards models. Non-parametric methods have the advantages of no assumptions for the underlying survival distributions; while Cox model made certain assumptions about the nature of hazard function for proportional hazards regression method. In practice, it can be assumed that the survival function is of a certain form such as exponential, Weibull, and so on, with one or more parameters whose values are unknown, to be estimated from real data [58]. Furthermore, if the shape of the survival distribution is known, parametric regression models may produce more efficient estimates than Cox model [59].

Till now, the sample sizes used in miRNA expression studies are relatively small, therefore, Bayesian methods may have some advantages in flexibility and for incorporating information from previous studies. Future studies with large sample sizes will be required to increase the power in detection of the significance and also for adjusting for multiple covariates in regression models. In addition, due to the complex correlation structure between multiple tests, adjusting for multiple testing is a big statistical challenge but is essential in analysis of miRNA expression with human cancers.

\section{References}

1. Ambros $V$ (2004) The functions of animal microRNAs. Nature 431: 350-355.

2. Bartel DP (2004) MicroRNAs: genomics, biogenesis, mechanism, and function. Cell 116: 281-297.

3. Carthew RW, Sontheimer EJ (2009) Origins and Mechanisms of miRNAs and siRNAs. Cell 136: 642-655.

4. Lagos-Quintana M1, Rauhut R, Lendeckel W, Tuschl T (2001) Identification of novel genes coding for small expressed RNAs. Science 294: 853-858.

5. Ambros $\vee$ (2003) MicroRNA pathways in flies and worms: growth, death, fat, stress, and timing. Cell 113: 673-676.

6. Cummins JM, Velculescu VE (2006) Implications of micro-RNA profiling for cancer diagnosis. Oncogene 25: 6220-6227.

7. Slack FJ, Weidhaas JB (2006) MicroRNAs as a potential magic bullet in cancer. Future Oncol 2: 73-82.

8. Peter ME (2009) Let-7 and miR-200 microRNAs: guardians against pluripotency and cancer progression. Cell Cycle 8: 843-852.

9. Lu J, Getz G, Miska EA, Alvarez-Saavedra E, Lamb J, et al. (2005) MicroRNA expression profiles classify human cancers. Nature $435: 834-838$.

10. Kent OA, Mendell JT (2006) A small piece in the cancer puzzle: microRNAs as tumor suppressors and oncogenes. Oncogene 25: 6188-6196.

11. Galasso M, Sandhu SK, Volinia S (2012) MicroRNA expression signatures in solid malignancies. Cancer J 18: 238-243.

12. Lu Y, Govindan R, Wang L, Liu PY, Goodgame B, et al. (2012) MicroRNA profiling and prediction of recurrence/relapse-free survival in stage I lung cancer. Carcinogenesis 33: 1046-1054.

13. Chen C, Ridzon DA, Broomer AJ, Zhou Z, Lee DH, et al. (2005) Real-time quantification of microRNAs by stem-loop RT-PCR. Nucleic Acids Res 33 : e179

14. Shingara J, Keiger K, Shelton J, Laosinchai-Wolf W, Powers P, et al. (2005) An optimized isolation and labeling platform for accurate microRNA expression profiling. RNA 11: 1461-1470.

15. Buermans HP, Ariyurek Y, van Ommen G, den Dunnen JT, 't Hoen PA (2010) New methods for next generation sequencing based microRNA expression profiling. BMC Genomics 11: 716.

16. Barbano R, Palumbo O, Pasculli B, Galasso M, Volinia S, et al (2014) miRNA signature for defining aggressive phenotype and prognosis in gliomas. PLoS One 9: e108950.

17. Leidinger P, Backes C, Dahmke IN, Galata V, Huwer H, et al. (2014) What makes a blood cell based miRNA expression pattern disease specific?--a miRNome analysis of blood cell subsets in lung cancer patients and healthy controls. Oncotarget 5: 9484-9497.

18. Robinson MD, McCarthy DJ, Smyth GK (2010) edgeR: a Bioconducto package for differential expression analysis of digital gene expression data. Bioinformatics 26: 139-140.

19. Robinson MD, Oshlack A (2010) A scaling normalization method for differential expression analysis of RNA-seq data. Genome Biol 11: R25.

20. Livak KJ, Schmittgen TD (2001) Analysis of relative gene expression data using real-time quantitative PCR and the 2(-Delta Delta $\mathrm{C}(\mathrm{T})$ ) Method. Methods 25: 402-408.

21. Li X, Shi Y, Yin Z, Xue X, Zhou B1 (2014) An eight-miRNA signature as a potential biomarker for predicting survival in lung adenocarcinoma. $\mathrm{J}$ Trans Med 12: 159.

22. Wang XC, Wang W, Zhang ZB, Zhao J, Tan XG, et al. (2013) Overexpression of miRNA-21 promotes radiation-resistance of non-small cell lung cancer. Radiat Oncol 8: 146

23. Lv J, Xu L, Xu Y, Qiu M, Yang X, et al. (2014) [Expression of MiRNA-221 in non-small cell lung cancer tissues and correlation with prognosis]. Zhongguo Fei Ai Za Zhi 17: 221-225.

24. Xie C, Han Y, Liu Y, Han L, Liu J (2014) miRNA-124 down-regulates SOX8 expression and suppresses cell proliferation in non-small cell lung cancer. Int $J$ Clin Exp Pathol 7: 7518-7526.

25. Keller A, Leidinger $P$, Gislefoss $R$, Haugen A, Langseth $H$, et al. (2011) Stable serum miRNA profiles as potential tool for non-invasive lung cancer diagnosis. RNA Biol 8: 506-516.

26. Li J, Yang H, Li Y, Liu Y, Chen S, et al. (2014) microRNA-146 up-regulation predicts the prognosis of non-small cell lung cancer by miRNA in situ hybridization. Exp Mol Pathol 96: 195-199.

27. Meng W, Ye Z, Cui R, Perry J, Dedousi-Huebner V, et al. (2013) MicroRNA-31 predicts the presence of lymph node metastases and survival in patients with lung adenocarcinoma. Clin Cancer Res 19: 5423-5433.

28. Zhang X, Ni Z, Duan Z, Xin Z, Wang H, et al. (2015) Overexpression of E2F mRNAs associated with gastric cancer progression identified by the transcription factor and miRNA co-regulatory network analysis. PLoS One 10: e0116979.

29. Guo L, Zhao Y, Yang S, Cai M, Wu Q, et al. (2013) Genome-wide screen for aberrantly expressed miRNAs reveals miRNA profile signature in breast cancer. Mol Biol Rep 40: 2175-2186.

30. Lichner Z, Fendler A, Saleh C, Nasser AN, Boles D, et al. (2013) MicroRNA signature helps distinguish early from late biochemical failure in prostate cancer. Clin Chem 59: 1595-1603. 
Citation: Wang KS, Pan Y, Xu C (2015) Statistical Modeling of MicroRNA Expression with Human Cancers. J Biom Biostat 6: 240. doi:10.4172/21556180.1000240

31. Chen Y, Min L, Zhang X, Hu S, Wang B, et al. (2013) Decreased miRNA$148 \mathrm{a}$ is associated with lymph node metastasis and poor clinical outcomes and functions as a suppressor of tumor metastasis in non-small cell lung cancer. Oncol Rep 30: 1832-1840.

32. Van der Auwera I, Limame R, van Dam P, Vermeulen PB, Dirix LY, et al. (2010) Integrated miRNA and mRNA expression profiling of the inflammatory breast cancer subtype. Br J Cancer 103: 532-541.

33. Khodyrev DS, Pronina IV, Rykov SV, Beresneva EV, Fridman MV, et al. (2012) [Methylation of some miRNA genes is involved in the regulation of their target genes RAR-beta2 and NKIRAS1 expression in lung cancer]. Mol Biol (Mosk) 46: 773-785.

34. Devor EJ, Schickling BM, Leslie KK (2014) microRNA expression patterns across seven cancers are highly correlated and dominated by evolutionarily ancient families. Biomed Rep 2: 384-387.

35. Boren T, Xiong Y, Hakam A, Wenham R, Apte S, et al. (2009) MicroRNAs and their target messenger RNAs associated with ovarian cancer response to chemotherapy. Gynecol Oncol 113: 249-255

36. Landi MT, Zhao Y, Rotunno M, Koshiol J, Liu H, et al. (2010) MicroRNA expression differentiates histology and predicts survival of lung cancer. Clin Cancer Res 16: 430-441.

37. Wang F, Wong SC, Chan LW, Cho WC, Yip SP, et al. (2014) Multiple regression analysis of mRNA-miRNA associations in colorectal cancer pathway. Biomed Res Int 2014: 676724.

38. De Preter K1, Mestdagh P, Vermeulen J, Zeka F, Naranjo A, et al. (2011) miRNA expression profiling enables risk stratification in archived and fresh neuroblastoma tumor samples. Clin Cancer Res 17: 7684-7692.

39. McDermott AM, Miller N, Wall D, Martyn LM, Ball G, et al. (2014) Identification and validation of oncologic miRNA biomarkers for luminal A-like breast cancer. PLoS One 9: e87032.

40. Hanson EK, Mirza M, Rekab K, Ballantyne J (2014) The identification of menstrual blood in forensic samples by logistic regression modeling of miRNA expression. Electrophoresis 35: 3087-3095.

41. Zhang Y, Sturgis EM, Sun Y, Sun C, Wei Q, et al. (2015) A functional variant at miRNA-122 binding site in IL-1 $1 \pm$ 3' UTR predicts risk and HPV-positive tumours of oropharyngeal cancer. Eur J Cancer 51: 1415-1423.

42. Wang ZX, Bian HB, Wang JR, Cheng ZX, Wang KM, et al. (2011) Prognostic significance of serum miRNA-21 expression in human non-small cell lung cancer. J Surg Oncol 104: 847-851.

43. Li R, Gao K, Luo H, Wang X, Shi $Y$, et al. (2014) Identification of intrinsic subtype-specific prognostic microRNAs in primary glioblastoma. J Exp Clin Cancer Res 33: 9.

44. Mankoo PK, Shen R, Schultz N, Levine DA, Sander C (2011) Time to recurrence and survival in serous ovarian tumors predicted from integrated genomic profiles. PLoS One 6: e24709.

45. Goeman JJ, van de Geer SA, de Kort F, van Houwelingen HC (2004) A global test for groups of genes: testing association with a clinical outcome. Bioinformatics 20: 93-99.

46. Oberg AL, French AJ, Sarver AL, Subramanian S, Morlan BW, et al. (2011) miRNA expression in colon polyps provides evidence for a multihit model of colon cancer. PLoS One 6: e20465.

47. Stephens M, Balding DJ (2009) Bayesian statistical methods for genetic association studies. Nat Rev Genet 10: 681-690.

48. Efron B (2010) Large-Scale Inference: Empirical Bayes Methods for Estimation Testing, and Prediction. Cambridge University Press, New York.

49. Witten DM, Tibshirani R (2010) A framework for feature selection in clustering J Am Stat Assoc 105: 713-726.

50. Wang W, Baladandayuthapani V, Morris JS, Broom BM, Manyam G, et al (2013) iBAG: integrative Bayesian analysis of high-dimensional multiplatform genomics data. Bioinformatics 29: 149-159.

51. Srivastava S, Wang W, Manyam G, Ordonez C, Baladandayuthapani V (2013) Integrating multi-platform genomic data using hierarchical Bayesian relevance vector machines. EURASIP J Bioinform Syst Biol 2013: 9.

52. Chekouo T, Stingo FC, Doecke JD, Do KA (2015) miRNA-target gene regulatory networks: A Bayesian integrative approach to biomarker selection with application to kidney cancer. Biometrics 71: 428-438.

53. Lam TK, Shao S, Zhao Y, Marincola F, Pesatori A, et al. (2012) Influence of quercetin-rich food intake on microRNA expression in lung cancer tissues. Cancer Epidemiol Biomarkers Prev 21: 2176-2184.

54. Benjamini $Y$, Hochberg $Y(1995)$ Controlling the false discovery rate - a practica and powerful approach to multiple testing. J Royal Stat Soc SeriesB-Methodol 57: 289-300.

55. Rahmann S, Martin M, Schulte JH, Köster J, Marschall T, et al. (2013) Identifying transcriptional miRNA biomarkers by integrating high-throughput sequencing and real-time PCR data. Methods 59: 154-163.

56. Camps C, Saini HK, Mole DR, Choudhry H, Reczko M, et al. (2014) Integrated analysis of microRNA and mRNA expression and association with HIF binding reveals the complexity of microRNA expression regulation under hypoxia. Mol Cancer 13: 28.

57. Skrzypski M, Czapiewski P, Goryca K, Jassem E, Wyrwicz L, et al. (2014) Prognostic value of microRNA expression in operable non-small cell lung cancer patients. Br J Cancer 110: 991-1000.

58. Cantor AB (2007) SAS Survival Analysis Techniques for Medical Research (3rdedn.) SAS institute INC, Cary, NC.

59. Allison PD (2010) Survival Analysis Using SAS: A Practical Guide (2ndedn.) SAS Institute inc., Cary, NC. 